\title{
Proximal Scaphoid Arthroplasty Using the Medial Femoral Trochlea Flap
}

\author{
James P. Higgins, MD ${ }^{1}$ Heinze K. Burger, MD² \\ ${ }^{1}$ The Curtis National Hand Center, MedStar Union Memorial Hospital, \\ Baltimore, Maryland \\ 2 Privat Hospital Maria Hilf, Klagenfurt, Austria
}

J Wrist Surg 2013;2:228-233.

\begin{abstract}
Address for correspondence James P. Higgins, MD, Care of Anne Mattson, The Curtis National Hand Center, MedStar Union Memorial Hospital, 3333 North Calvert Street, Mezzanine, Baltimore, MD 21218 (e-mail: anne.mattson@medstar.net).
\end{abstract}

\begin{abstract}
Keywords

- medial femoral condyle

- medial femoral trochlea flap

- osteocartilaginous autograft

- scaphoid nonunion

- vascularized bone

Background The medial trochlea of the femur (medial femoral trochlea, MFT) provides a source of convex osteocartilaginous vascularized bone that has been demonstrated to have a similar contour to the proximal scaphoid. This provides a potential solution for difficult recalcitrant proximal pole scaphoid nonunions.

Materials and Methods Sixteen consecutive patients who underwent MFT proximal scaphoid arthroplasty were reviewed. Follow-up data were recorded at a minimum of 6 months, with an average of 14 months. The results of this cohort were previously reported in detail but are summarized herein.

Description of Technique The ability to reconstruct both bone and cartilage of the nonunion enables the surgeon to resect the nonunited proximal pole to prepare for scaphoid reconstruction. A segment of osteocartilaginous MFT is harvested in dimensions required by the scaphoid defect. The MFT segment is harvested on the transverse branch of the descending geniculate vessels. Fixation may be achieved with ease due to the size of the reconstructed segment.

Results Computed tomography imaging demonstrated 15 of 16 reconstructed scaphoids achieving osseous union. Follow-up range of motion (ROM) of the wrist averaged $46.0^{\circ}$ extension (range $28-80^{\circ}$ ) and $43.8^{\circ}$ flexion (range $10-80^{\circ}$ ), which was similar to preoperative (average $45.7^{\circ}$ extension and $43.0^{\circ}$ flexion). Scapholunate angles remained unaffected $\left(51.6^{\circ}\right.$ preoperatively and $48.6^{\circ}$ postoperatively), indicating preservation of carpal relationships.

Conclusions Vascularized MFT flaps provide a useful tool in the treatment of difficult proximal pole scaphoid nonunions. Early follow-up demonstrates high rate of achieving union with acceptable ROM and good pain relief.
\end{abstract}

Treatment of the proximal pole scaphoid nonunion presents many challenges to the reconstructive surgeon. Conventional treatment options include achievement of rigid internal fixation with bone grafting, local vascularized pedicle bone flaps, or microvascular free bone flaps. Longstanding scaphoid nonunion can lead to a progressive and predictable pattern of wrist arthritis, requiring undesirable surgical salvage procedures.

The recalcitrant nonunion of the proximal pole will often present with a very small and poor-quality proximal segment after multiple previous surgical fixation attempts. It is in this setting that conventional surgical treatment options provide inadequate solutions for the absence or poor quality of proximal pole cartilage.

The medial femoral condyle (MFC) has been recognized as a useful source of vascularized bone for treatment of scaphoid nonunions. ${ }^{1,2}$ The vascular supply to the surrounding region of the distal femur has been well described. ${ }^{3-6}$ The descending genicular arterial vascular system provides a dense filigree of blood vessels invested in the periosteum of the medial
Copyright @ 2013 by Thieme Medical Publishers, Inc., 333 Seventh Avenue, New York, NY 10001, USA. Tel: +1(212) 584-4662.
DOI http://dx.doi.org/ 10.1055/s-0033-1351789. ISSN 2163-3916. 
column of the distal femur. The descending genicular artery (DGA) divides into two major branches within the periosteum: the longitudinal branch and the transverse branch. The longitudinal branch courses to the condyle and is used as the source vessel for the corticoperiosteal flap commonly used for scaphoid nonunions. The transverse branch of the DGA courses anteriorly, investing in the periosteum surrounding the medial femoral trochlea (MFT) ${ }^{5,6}$ This site has been observed to be a source of a convex cartilaginous flap with a curvature similar to that of the proximal scaphoid as well as the concave surface of the scaphoid fossa. ${ }^{6}$ The use of this segment of bone and cartilage as a vascularized flap for proximal scaphoid reconstruction was initially described by the senior author (HKB) in a case report in 2008. ${ }^{7}$ Subsequently, we have reported our early experience in the use of this flap for scaphoid nonunion, resulting in good intermediate-term results. ${ }^{8}$ This article will report our experience with this flap for the treatment of recalcitrant nonunions and describe the technique.

\section{Patients and Methods}

The authors reviewed the initial 16 consecutive patients who underwent MFT flap osteocartilaginous arthroplasty of the proximal scaphoid in their two surgical practices. The authors performed these procedures between January 2006 and February 2012 on patients with established proximal pole nonunions. Scaphoid nonunions were defined as those greater than 6 months of attempted fracture treatment by either closed or open technique. Patients were considered candidates for osteocartilaginous arthroplasty using the MFT flap if the remaining proximal pole cartilaginous shell was considered too poor to permit conventional reconstruction and fixation. These patients were all confirmed to have no evidence of radiographic arthritis. Six patients had undergone previous fixation attempts with vascularized bone grafting procedures, four patients had undergone previous screw fixation without bone grafting, and one patient had undergone previous surgical treatment with nonvascularized bone grafting. The remaining five patients had not undergone any previous surgical intervention. These patients all had extremely small proximal pole fragments that were diagnosed as having avascular necrosis on preoperative magnetic resonance imaging (MRI). These patients all were referred with MRI studies. The authors do not consider MRI evidence of avascular necrosis as a prerequisite for MFT reconstruction.

The patient charts were reviewed for the following data: age, gender, and duration of nonunion, surgical approach (volar/dorsal), previous surgical procedures, method of fixation, achievement of healing, preoperative and postoperative range of motion (ROM), scapholunate angles, and relief of pain.

\section{Surgical Technique}

The surgical approach for harvesting MFC flap and MFT flap through the soft tissue is identical. Iorio et al. have described this surgical approach, including the potential use of a skin segment of the flap in its harvesting technique, elsewhere. ${ }^{9}$ The flap can be harvested with a skin segment overlying the medial aspect of the knee, based on a cutaneous branch from the DGA pedicle. This would be performed to permit surface monitoring of the flap viability as well as permit ease of closure of the skin over the anastomosis. Alternatively, the surgeon may elect to monitor the anastomosis with an implantable Doppler monitor. Skin closure can be achieved with mobilization of local skin flaps or skin grafts if needed.

The DGA can be traced distally along the medial column of the femur to feed a broad filigree of blood vessels intimately adherent into the periosteum of the medial distal femur (-Fig. 1). The longitudinal branch supplies the region commonly used for harvest of the MFC osteoperiosteal flap. The perpendicular transverse branch typically traverses the metaphyseal region and densely supplies the periosteum surrounding the MFT both on its medial aspect and proximal aspect. This is the vessel that is utilized in this case.

Prior to harvesting the flap, the wrist dissection is prepared. The reconstruction may be performed via a volar or dorsal approach, according to the surgeon's preference. The dorsal approach provides greater ease of visualization of the scaphoid fossa and the nearby snuffbox for microvascular anastomosis. The volar approach can be performed through a less extensive exposure and permits screw placement from distal to proximal. In this approach the arterial anastomosis could be performed via an end-to-side anastomosis into the radial artery or end-to-end into the palmar branch of the radial artery.

With either approach, the surgeon must confirm the absence of arthritis in the scaphoid fossa. If the scaphoid fossa is deemed adequate to proceed with reconstruction, the proximal pole nonunion segment is resected. This resection is performed carefully with three objectives ( - Fig. 2). First, the thin cartilaginous surface that effaces the midcarpal joint is meticulously preserved. The MFT flap will provide a cancellous surface facing distally and will require the native scaphoid cartilage to maintain the smooth articulation with the

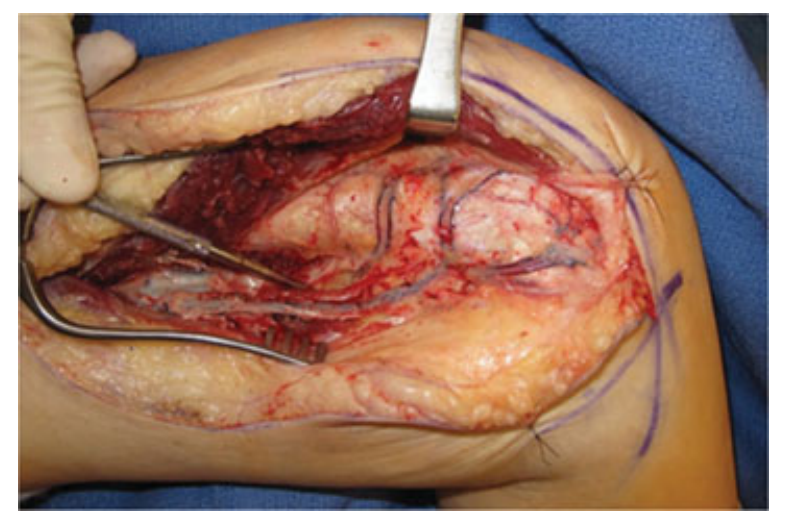

Fig. 1 Medial view of femur after elevation of the vastus medialis. Longitudinal coursing DGA vessel is seen running from superficial femoral artery on left to MFC on right. Note the network of vessels on the medial femur, including the longitudinal vessel directed to the condyle and the transverse branch running toward the patellofemoral joint. This exposure is much more extensive than that required for the scaphoid reconstruction, but it is provided to demonstrate the vascular anatomy of the DGA system. 


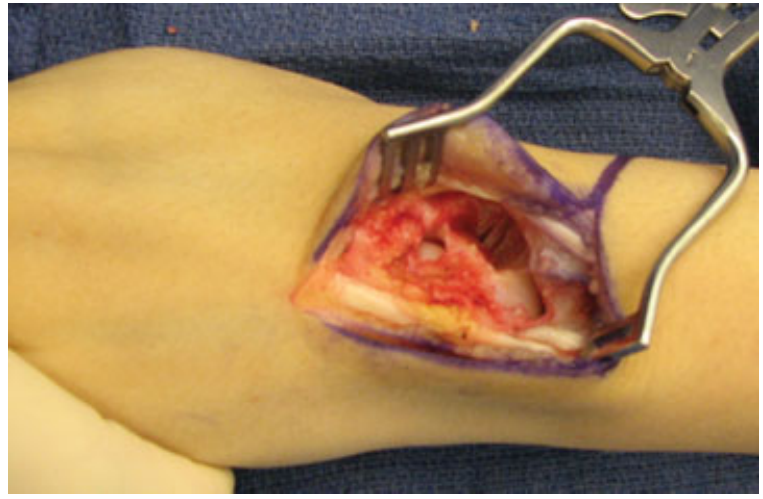

Fig. 2 View of dorsal approach to scaphoid nonunion after resection of proximal pole and nonunion site in preparation for reconstruction. Fingers are to the left and forearm to the right. Note the generous resection and the preservation of the midcarpal articulating cartilage.

capitate. Second, the most distal aspects of the dorsal and volar scapholunate ligaments are preserved, in continuity with the distal rim of cartilage. This is done to provide a benefit of stability between the scaphoid and the lunate and inhibit rotary instability of the scaphoid. Third, the débridement of the proximal pole segment extends well beyond the nonunion plane and includes a significant amount of the distal segment. The MFT flap provides a larger osteocartilaginous segment that permits creation of a defect large enough to permit ease of inset. Creating a larger defect and larger flap provides the surgeon with greater ease of fixation as well as the ability to provide an uninterrupted smooth cartilage surface for articulation with the scaphoid fossa. The preparation of the defect thus converts the difficult proximal pole fracture into a more manageable scaphoid waist osteosynthesis.
With attention turned to the knee and the soft tissue dissection completed, the DGA is visualized. It may be sutureligated proximally from its origin off the superficial femoral artery within the adductor canal. This will yield a lengthy pedicle beyond the requirements of the reconstruction. This length of pedicle is desirable only in that it will provide the greatest caliber point of the DGA for microvascular anastomosis.

After the DGA is ligated from the superficial femoral artery, the DGA is reflected distally. Smaller branches are ligated as the dissection approaches the distal femur. As the DGA becomes intimately embedded in the periosteum, the two larger branches will become apparent. The longitudinal branch to the condyle may be suture-ligated. Harvest of vessel continues using the transverse branch that can be traced toward the medial trochlea. As the vessels become progressively smaller, the dissection is performed subperiosteally so as not to injure the small artery or veins. The periosteal vessels are left adherent to the territory of bone and cartilage desired for harvest. A sagittal saw and osteotomes can be used to harvest the most proximal portion of the cartilage-bearing segment of the MFT. The dimensions correspond as follows:

1. The proximal-to-distal harvest is measured to equal the radial-to-ulnar dimensions of the defect in the wrist.

2. The anterior-to-posterior harvest is measured to equal the proximal-to-distal dimension of the defect in the wrist.

3. The medial-to-lateral harvest in the knee is measured to equal the volar-to-dorsal dimensions of the wrist.

The segment is carefully harvested to be in close approximation to the measured defect in the wrist. After the flap is elevated, the capsule of the knee is closed, followed by layered

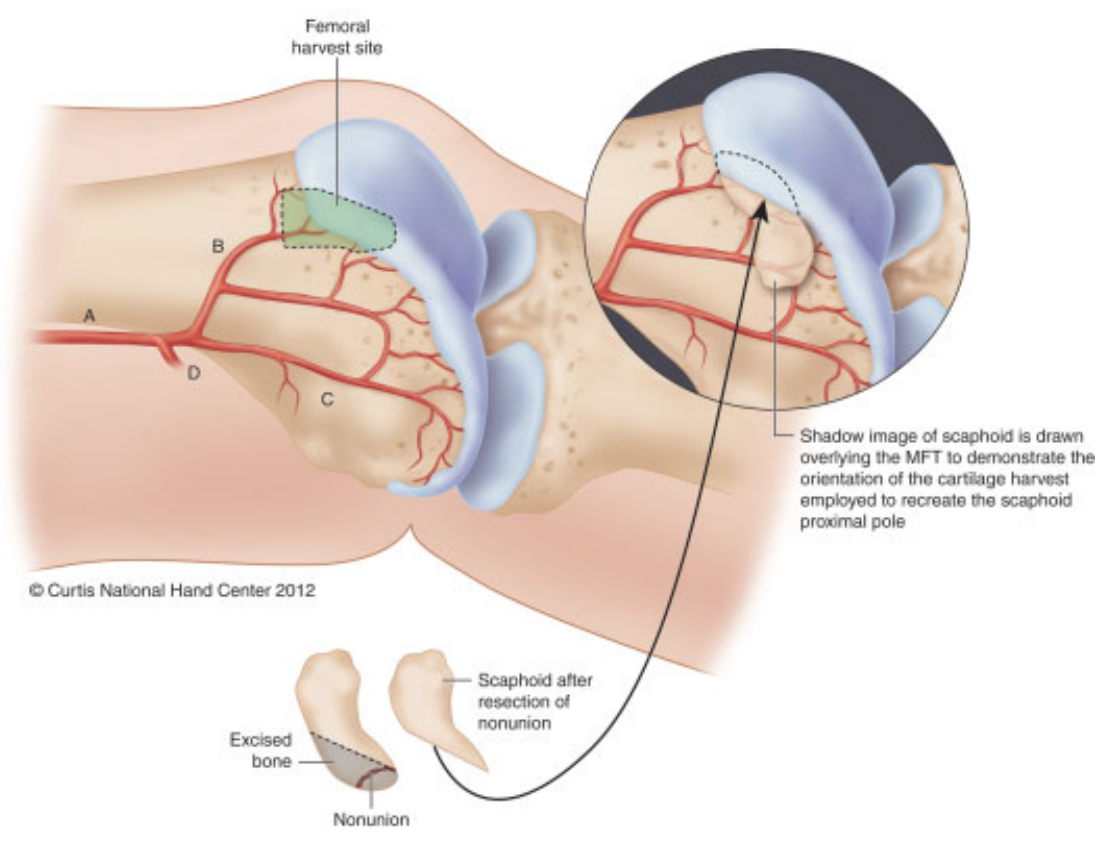

Fig. 3 Representation of MFT and the planned portion of reconstructed proximal scaphoid. Portion of MFT harvested to provide vascularized osteocartilaginous reconstruction of the proximal scaphoid. A, descending geniculate artery; B, transverse branch; C, longitudinal branch; D, superiomedial geniculate artery. (From Bürger HK, Windhofer C, Gaggl AJ, Higgins JP: Vascularized medial femoral trochlea osteocartilaginous flap reconstruction of proximal pole nonunions. J Hand Surg Am 2013 April; 38(4):690-700). 
closure of the donor site defect over a drain placed via counterincision.

The osteocartilaginous segment is then fashioned so as to fit as precisely as possible into the defect created from resection of the proximal scaphoid (-Fig. 3 ). The convex surface of the flap effaces the concave scaphoid fossa of the radius. This leaves a cancellous portion effacing the adjacent lunate as well as the preserved distal cartilaginous shell of the lesser curvature of the native scaphoid. A bone segment is usually affixed with a single cannulated screw driven from the dorsal or volar approach (surgeon's preference). Intraoperative fluoroscopy is utilized to confirm satisfactory reconstruction of the scaphoid and hardware placement.

Attention is then turned to the microsurgical anastomosis. When the approach is performed dorsally, the anastomosis is usually performed in the end-to-side fashion to the radial artery in the snuff box and associated veins. When the approach is performed volarly, the arterial anastomosis is usually performed in an end-to-end fashion into the palmar branch of the radial artery, with venous anastomosis into either deeper superficial vein in that region. If the skin can be closed without excessive tension, primary closure is performed. If excessive tension of closure demonstrates compression of the vessels, a small skin graft may be used. If the surgeon elects to harvest the flap with a small skin flap component, it will permit ease of tension-free closure as well as a means of monitoring the flap postoperatively.

Postoperatively, the patient's immobilization and radiographic monitoring would be similar to that of other conventional means of scaphoid nonunion surgery. The patient is permitted to ambulate immediately after surgery. It is common for the patient to have some discomfort with ambulation that will resolve over the postoperative 2 to 4 months. No knee brace or other immobilization of the knee is required.

\section{Results}

The results of this cohort were previously reported in detail ${ }^{8}$ but are summarized here as follows. Healing in the cohort of 16 consecutive osteocartilaginous scaphoid arthroplasties was confirmed by CT scan in 15 patients. In one patient, healing was not achieved, and the patient is considering salvage operations.
A minimum of 6 months follow up was required for inclusion in this series. The average follow up was 14 months with a range from 6 to 72 months. The median age of the patients was 30 years with a range of 18 to 47 years. The average number of previous operations was 1 with a range of 0 to 3 . Seven of 16 patients were smokers.

The surgical technique varied for the 16 cases, with 13 of 16 being approached volarly and the remaining 3 being approached dorsally. Six cases were treated with cannulated screw fixation, four with screw and mini-plate combination, three with mini-plate and Kirschner wire (K-wire), two with K-wires alone, and one with mini-plate alone.

Of the patients, 12 of 16 reported complete pain relief, while 4 of 16 reported improvement without complete pain relief. Pronosupination and digital ROM were unaffected when comparing preoperative to postoperative values. Preoperative extension averaged $45.7^{\circ}$, and postoperative extension averaged $46^{\circ}$. Preoperative flexion averaged $43^{\circ}$, and postoperative flexion averaged $43.8^{\circ}$.

Radiographically, the analysis of the intercarpal relationships demonstrated relatively unchanged scapholunate angles, with an average of $51.6^{\circ}$ preoperatively and $48.6^{\circ}$ postoperatively.

\section{Discussion}

The DGA system has demonstrated versatility as both a donor and recipient site for microvascular reconstruction. In upper extremity surgery it has been shown to be of use as a pliable corticoperiosteal flap, ${ }^{10,11}$ a corticocancellous semistructural flap, ${ }^{12-17}$ and a skin-bearing flap. ${ }^{3,9,12,14,16,18-21}$ Its transverse branch has been well studied and noted to provide a periosteal filigree of vessels that supply the cartilage-bearing area of the proximal aspect of the medial trochlea of the patellofemoral joint. The osteology of this convex surface has been observed and demonstrated to be of similar contour and curvature as the greater arc of the scaphoid as well as the mirror image scaphoid fossa. ${ }^{6}$

These qualities have made the MFT flap a useful tool in treating recalcitrant proximal pole nonunions. Its indication for use is in young patients with scaphoid nonunions demonstrating a very small proximal pole fracture fragment that has failed at previous attempts at reconstruction (-Fig. 4). In
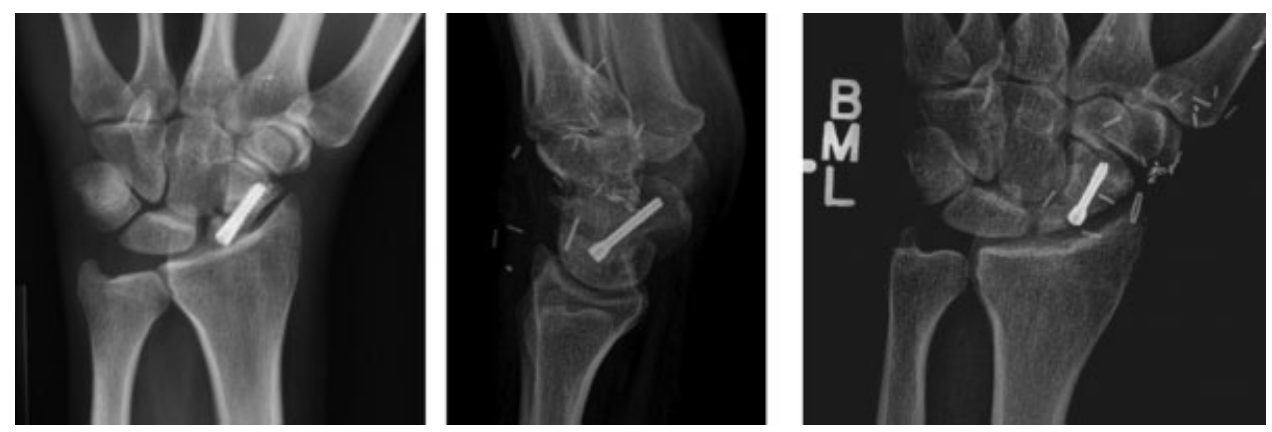

Fig. 4 Preoperative anteroposterior (AP) (left) and postoperative AP and lateral (right) radiographs of MFT reconstruction of proximal pole nonunion. Note the preservation of the scapholunate $(\mathrm{SL})$ angle. The cartilage layer of the MFT flap is 3-5 mm thick, often making the trailing screws of a dorsally placed screw appear prominent. 
these situations, conventional reconstructive techniques cannot provide both improved vascularity as well as rigid internal fixation. When the proximal fragment is extremely small, and particularly when it has been previously damaged by single or multiple attempts at screw fixation, it is particularly difficult to achieve adequate fixation because conventional vascularized or nonvascularized correction procedures require preservation of this damaged cartilage shell. The MFT flap provides the surgeon with the ability to resect this damaged fragment widely and resect distally into the normal scaphoid fragment, enlarging the nonunion site and converting the proximal pole fracture to a waist-level defect. This larger defect can be filled because of the ability to transfer this morphologically similar convex osteocartilaginous flap. The larger sizes of the flap enable us to preserve a generous vascular pedicle securely and achieve rigid internal fixation easily on both proximal and distal fragments. These attributes enable the surgeon to deliver both improved vascularity and fixation to difficult nonunion cases and achieve union despite previous failures. It also enables the surgeon to make a challenging technical case somewhat more feasible by supplying fixation and not feeling compelled to preserve a damaged and compromised cartilaginous shell.

Our greatest concern with this technique was the ability to maintain intercarpal relationships after resecting the majority of the scapholunate ligament and proximal pole. We take great effort to preserve the most distal cartilaginous shell of the native scaphoid that effaces the midcarpal joint, as well as the most distal aspects of the dorsal scapholunate ligament. The case series demonstrated good preservation of the scapholunate angle at intermediate follow-up. ${ }^{8}$ The majority of our patients demonstrated slight decrease in their scapholunate angles. This suggests clinically that the preservation of the most distal scapholunate ligaments may be adequate to maintain the relationship between the healed scaphoid and the lunate.

Another great concern is that of donor site morbidity. These patients will typically report transient discomfort in the knee after MFT harvest, but they have been able to return to normal ambulation as well as sporting activities without difficulty. Many of these patients are following up in very demanding athletic endeavors and have all been able to return to preinjury activities. We will continue to follow these patients for long-term follow-up and plan to reassess radiographic and subjective outcomes in the donor site.

Although these findings are quite promising for addressing this very difficult problem of recalcitrant proximal pole scaphoid nonunions, there are many unaddressed questions. We will continue to follow these patients and report on longterm radiographic and subjective outcomes so that we may better determine the value of this reconstructive option and its ability to preserve function and avoid arthritic changes. Further basic research is also warranted to determine the relative value of periosteal and subchondral vascular supply to cartilage in relation to that of synovial perfusion.

The MFT osteocartilaginous flap provides a promising new tool for treatment of difficult recalcitrant proximal pole scaphoid nonunions. Early follow-up demonstrates a high rate of success in achieving osseous union, good preservation of intercarpal relationships, and good preservation of wrist ROM and resolution or improvement of pain.

\section{Note}

The work was performed at both of the authors' locations.

\section{Conflict of Interest \\ None}

\section{References}

1 Doi K, Oda T, Soo-Heong T, Nanda V. Free vascularized bone graft for nonunion of the scaphoid. J Hand Surg Am 2000;25(3): 507-519

2 Jones DB Jr, Bürger H, Bishop AT, Shin AY. Treatment of scaphoid waist nonunions with an avascular proximal pole and carpal collapse. Surgical technique. J Bone Joint Surg Am 2009; 91(Suppl 2):169-183

3 Martin D, Bitonti-Grillo C, De Biscop J, et al. Mandibular reconstruction using a free vascularised osteocutaneous flap from the internal condyle of the femur. Br J Plast Surg 1991;44(6):397-402

4 Yamamoto H, Jones DB Jr, Moran SL, Bishop AT, Shin AY. The arterial anatomy of the medial femoral condyle and its clinical implications. J Hand Surg Eur Vol 2010;35(7):569-574

5 Iorio ML, Masden DL, Higgins JP. The limits of medial femoral condyle corticoperiosteal flaps. J Hand Surg Am 2011;36 (10):1592-1596

6 Hugon S, Koninckx A, Barbier O. Vascularized osteochondral graft from the medial femoral trochlea: anatomical study and clinical perspectives. Surg Radiol Anat 2010;32(9):817-825

7 Kälicke T, Bürger H, Müller EJ. A new vascularized cartilage-bonegraft for scaphoid nonunion with avascular necrosis of the proximal pole. Description of a new type of surgical procedure [in German]. Unfallchirurg 2008;111(3):201-205

8 Bürger HK, Windhofer C, Gaggl AJ, Higgins JP. Vascularized medial femoral trochlea osteocartilaginous flap reconstruction of proximal pole scaphoid nonunions. J Hand Surg Am 2013;38(4): 690-700

9 Iorio ML, Masden DL, Higgins JP. Cutaneous angiosome territory of the medial femoral condyle osteocutaneous flap. J Hand Surg Am 2012;37(5):1033-1041

10 Fuchs B, Steinmann SP, Bishop AT. Free vascularized corticoperiosteal bone graft for the treatment of persistent nonunion of the clavicle. J Shoulder Elbow Surg 2005;14(3):264-268

11 Kaminski A, Bürger H, Müller EJ. Free vascularised corticoperiosteal bone flaps in the treatment of non-union of long bones: an ignored opportunity? Acta Orthop Belg 2008;74(2):235-239

12 Del Piñal F, García-Bernal FJ, Regalado J, Ayala H, Cagigal L, Studer A. Vascularised corticoperiosteal grafts from the medial femoral condyle for difficult non-unions of the upper limb. J Hand Surg Eur Vol 2007;32(2):135-142

13 Sammer DM, Bishop AT, Shin AY. Vascularized medial femoral condyle graft for thumb metacarpal reconstruction: case report. J Hand Surg Am 2009;34(4):715-718

14 Pelzer M, Reichenberger M, Germann G. Osteo-periosteal-cutaneous flaps of the medial femoral condyle: a valuable modification for selected clinical situations. J Reconstr Microsurg 2010;26 (5):291-294

15 Grant I, Berger AC, Ireland DC. A vascularised bone graft from the medial femoral condyle for recurrent failed arthrodesis of the distal interphalangeal joint. Br J Plast Surg 2005;58(7): 1011-1013 
16 Gaggl A, Bürger H, Chiari FM. The microvascular osteocutaneous femur transplant for covering combined alveolar ridge and floor of the mouth defects: preliminary report. J Reconstr Microsurg 2008;24(3):169-175

17 Gaggl AJ, Bürger HK, Chiari FM. Free microvascular transfer of segmental corticocancellous femur for reconstruction of the alveolar ridge. Br J Oral Maxillofac Surg 2008;46(3):211-217

18 Doi K, Sakai K. Vascularized periosteal bone graft from the supracondylar region of the femur. Microsurgery 1994;15(5):305-315
19 Sakai K, Doi K, Kawai S. Free vascularized thin corticoperiosteal graft. Plast Reconstr Surg 1991;87(2):290-298

20 Kobayashi S, Kakibuchi M, Masuda T, Ohmori K. Use of vascularized corticoperiosteal flap from the femur for reconstruction of the orbit. Ann Plast Surg 1994;33(4):351-357, discussion 357-358

21 Rahmanian-Schwarz A, Spetzler V, Amr A, Pfau M, Schaller HE, Hirt B. A composite osteomusculocutaneous free flap from the medial femoral condyle for reconstruction of complex defects. J Reconstr Microsurg 2011;27(4):251-260 